\title{
Prevalence of gyrA and $B$ gene mutations in fluoroquinolone-resistant and -sensitive clinical isolates of Mycobacterium tuberculosis and their relationship with MIC of ofloxacin
}

\begin{abstract}
Pooja Singh, Amita Jain, Pratima Dixit, Shantanu Prakash, Indu Jaiswal, Vimala Venkatesh and Mastan Singh
The study was done to know the prevalent mutations of gyrA and gyrB genes, and their significance with drug resistance in clinical isolates of Mycobacterium tuberculosis. A total of 100 ofloxacin- (OFX) resistant and 100 OFX-sensitive isolates of M. tuberculosis were consecutively selected from routine Tuberculosis laboratory. Drug resistance pattern of these isolates was recorded. MIC of OFX was tested in all these isolates by absolute concentration method. Quinolone resistance determining region (QRDR) of gyrA and gyrB genes of 320 and $428 \mathrm{bp}$, respectively, were amplified and sequenced. Sequencing data were analyzed by BLAST on NCBI with reference strain H37Rv. Of 100 OFX-sensitive isolates, 30 were pansusceptible, 28 were monoresistant, 10 were polyresistant and 32 were multidrug resistant (MDR). Among 100 OFX-resistant isolates, 19 were OFX monoresistant, 16 were polyresistant and 65 were MDR. Mutations in gyrA and gyr $B$ genes were observed in $79 \%$ and $5 \%$ of OFX-resistant isolates, respectively. Most prevalent mutation was found at codon 94 in QRDR of gyrA gene. Double mutations found in gyrA gene and in both gyrA and gyrB genes signifies higher levels of OFX resistance. In one isolate, a substitution at codon 592 (Pro592Ser) was found as a novel mutation outside the QRDR of gyrB gene. Our findings support previous studies that the OFX resistance to M. tuberculosis is associated with mutations in the QRDR of gyrA gene; however, the level of OFX resistance may not be predicted based on the mutation patterns in the gyrA gene.
\end{abstract}

The Journal of Antibiotics (2015) 68, 63-66; doi:10.1038/ja.2014.95; published online 23 July 2014

Emergence of fluoroquinolone (FQ) resistance in Mycobacterium tuberculosis is due to the increasing numbers of FQ prescriptions, and the expanded use of these broad-spectrum agents for many infections leads to selective FQ pressure. In M. tuberculosis, FQs target DNA gyrase, ${ }^{1}$ which consist of two A and two B subunits encoded by the gyrA and gyrB genes, respectively. ${ }^{2}$ The quinolone resistance determining region (QRDR) is a short conserved region within the gyrA (codon 74-113) and gyrB (codon 500-538) $)^{1}$ genes. Studies have reported that the majority (approximately 50-90\%) of FQ-resistant M. tuberculosis isolates carry mutations in the QRDR of the gyrA gene, $^{3,4}$ and a small number $(7 \%)$ have mutations in the $g y r B$ gene. ${ }^{5}$ However, the genetic involvement of some gyrA and most gyrB gene mutations to FQ resistance in $M$. tuberculosis is not known. Therefore, this study was planned to identify mutations in the gyrA and $g y r B$ genes of $M$. tuberculosis and assessed their significance in determining the level of FQ resistance.

A total of 100 OFX-resistant and 100 OFX-sensitive isolates of M. tuberculosis were consecutively enrolled from the tuberculosis laboratory, Department of Microbiology, King George's Medical University, Lucknow, during August 2012 to July 2013. The drug resistance pattern of five antitubercular drugs streptomycin (SM), isoniazid (INH), rifampicin (RIF), ethambutol (ETH) and ofloxacin (OFX) were recorded. Laboratory quality control for drug susceptibility testing against SM, INH, RIF and EMB by the $1 \%$ proportion method is provided by the National JALMA Institute of Leprosy and Other Mycobacterial Diseases, Agra (India).

The OFX MICs were determined in all of these isolates by the absolute concentration method on Lowenstein Jensen (LJ) slants ${ }^{6}$ using a range of $1-16 \mu \mathrm{g} \mathrm{ml}^{-1}$. The standard strain of $M$. tuberculosis $\mathrm{H} 37 \mathrm{Rv}$ was tested as control (sensitive at $<1 \mu \mathrm{g} \mathrm{ml}^{-1}$ levels of OFX). Resistance to OFX was defined as MIC of $>2 \mu \mathrm{g} \mathrm{ml}^{-1}$. DNA extraction from M. tuberculosis isolates was done. ${ }^{7}$ The QRDR containing $320 \mathrm{bp}$ of the $\operatorname{gyr} A$ and the $428 \mathrm{bp}$ of $\operatorname{gyrB}$ gene was amplified. ${ }^{2}$ PCR products were analyzed by electrophoresis on $2 \%$ agarose gel. PCR products of the gyrA and $g y r B$ genes were sequenced using forward and reverse primers on an ABI Prism 3100 genetic analyzer (Applied Biosystems, Foster City, CA, USA) using BigDye Terminator chemistry (version 3.1) and analyzed by using BLAST with reference strain $M$. tuberculosis H37Rv. The DNA sequencing data reported in this study have been submitted to the NCBI database. The provided accession numbers are KC763836KC763837, KC777350-KC777357, KC819312-KC819320, KF760464- 
KF760514, KF826728-KF826739, KF826741-KF826744, KC880083KC880111， KF509920-KF509922, KF879517-KF879568 and KC819321-KC819341. SPSS software (SPSS, Chicago, IL, USA) was used for the statistical analysis. The MICs of OFX-resistant $M$. tuberculosis clinical isolates with different mutations in the gyrA gene were compared by the analysis of variance test.

Of the 100 OFX-sensitive isolates, 30 were pansusceptible, 28 were monoresistant, 10 were polyresistant and 32 were multidrug resistant (MDR). Monoresistance to INH was most common 12/28 (43\%). Among the $32 \mathrm{MDR}$ isolates, 11 (34.4\%) isolates were resistant to all four first-line antitubercular drugs. A total of 73 isolates had an OFX MIC value of $1 \mu \mathrm{g} \mathrm{ml}^{-1}$ and the remaining 27 isolates had an OFX MIC of $2 \mu \mathrm{g} \mathrm{ml}^{-1}$. No mutations in the $g y r A / B$ genes were detected in OFX-sensitive isolates, apart from a S95T mutation in the gyrA gene in 72 isolates, which was a natural polymorphism that occurred in both OFX-resistant and OFX-sensitive isolates in this study.

Among the 100 OFX-resistant isolates, 19 were OFX monoresistant, 16 were polyresistant and 65 were MDR. Of the 19 OFX monoresistant isolates, 2 had MICs of $4 \mu \mathrm{g} \mathrm{ml}^{-1}, 5 \mathrm{had}$ MICs of $8 \mu \mathrm{g} \mathrm{ml}^{-1}, 7$ had MICs of $16 \mu \mathrm{g} \mathrm{ml}^{-1}$ and 5 showed MICs $>16 \mu \mathrm{g} \mathrm{ml}^{-1}$. Of OFX monoresistant isolates, 17 (89.5\%) had mutations in the QRDR of the gyrA gene and no mutation was found in remaining 2 isolates. Among the 65 MDR isolates, 22 (34\%) isolates were panresistant, 23 (35.4\%) were resistant to SM, INH, RIF and OFX, $14(22 \%)$ were resistant to INH, RIF and OFX, and 6 (9.2\%) were resistant to INH, RIF, EMB and OFX. Nine MDR isolates had an OFX MICs of $4 \mu \mathrm{g} \mathrm{ml}^{-1}, 14 \mathrm{had}$ an OFX MICs of $8 \mu \mathrm{g} \mathrm{ml}^{-1}$, 35 had an OFX MICs of $16 \mu \mathrm{g} \mathrm{ml}^{-1}$ and 7 had an OFX MIC $>16 \mu \mathrm{g} \mathrm{ml}^{-1}$. Mutations in the QRDR of the gyrA gene were found in $51(78.5 \%)$ MDR isolates (Table 1).

Among OFX-resistant isolates, the S95T polymorphism was detected in 80 isolates. A total of $79(79 \%)$ isolates had mutations in the QRDR of the gyrA gene and $5(5 \%)$ isolates had mutations in the $g y r B$ gene, except the S95T mutation in the gyrA gene. In rest of the isolates no mutation was found in either $g y r A$ or $g y r B$ genes. Among the 79 isolates with the gyrA gene mutations, 68 (86.1\%) had single mutations and $11(14 \%)$ had double mutations in the QRDR. The most common single-nucleotide mutation sites were at codons 90 and 94 with a frequency of $24(35.3 \%)$ and 41 (60.1\%), respectively. The most frequent amino acid change (Asp94Gly) was found in 21/41 (51.2\%) isolates. Other mutations at codons 88 and 91 were also recorded. The OFX MICs of resistant isolates with mutations ranged from 4 to $>16 \mu \mathrm{g} \mathrm{ml}^{-1}$. Mutations in the gyrA gene and corresponding MICs of the 79 OFX-resistant isolates are shown in Table 2. Of OFX-resistant isolates with double substitutions $(n=11)$ in the gyrA gene, four $(36.5 \%)$ isolates had a combination of Ala90Val with Ser91Pro, three (27.3\%) had a combination of Ala90Val with Asp94Asn and three $(27.3 \%)$ had a combination of Ala90Val with Asp94Gly (Table 2). The OFX MICs in isolates with single mutations $(\mathrm{F}=1.36, P=0.26)$ and double mutations $(\mathrm{F}=0.25$, $P=0.78)$ in the $g y r A$ gene showed no significant difference.

Mutations in the gyrB gene were observed in 5 of the 100 OFXresistant isolates. The single-nucleotide mutation sites were in codons 500, 538, 539 (in two isolates) and 592. In one isolate, a substitution at codon 592 (Pro592Ser) was found as a novel mutation outside the QRDR of the gyrB gene. All of the isolates showing mutations in the gyrB gene also had mutations in the gyrA gene. No mutation was observed in the QRDR of the gyrB gene in OFX-sensitive isolates. The MIC of OFX-resistant isolates with mutations in the $\operatorname{gyr} B$ gene ranged from 8 to $>16 \mu \mathrm{g} \mathrm{ml}^{-1}$.

In the present study, an increase in frequency of OFX resistance was found. These results are consistent with data reported by earlier Indian studies ${ }^{8,9}$ and a study from Nigeria, ${ }^{10}$ but different than those reported in a study from Rawanda. ${ }^{11}$ In this study, no significant difference was found between the different mutation patterns in the gyrA gene with the level of OFX resistance in M. tuberculosis isolates, which is consistent with a study in East China. ${ }^{12}$ However, some studies have shown an association between mutations in the QRDR of

Table 1 Mutations detected in OFX-resistant $M$. tuberculosis isolates and their drug resistance pattern

\begin{tabular}{|c|c|c|c|c|c|c|c|c|c|c|c|}
\hline \multirow{2}{*}{$\begin{array}{l}\text { Drug resistance pattern of } \\
\text { OFX-resistant isolates }\end{array}$} & \multirow{2}{*}{$\begin{array}{c}\text { Total no. of } \\
\text { isolates }(\mathrm{n}=100)\end{array}$} & \multicolumn{4}{|c|}{$\begin{array}{c}\text { OFX MIC } \\
\left(\mu g m l^{-1}\right) \text { on } L J\end{array}$} & \multicolumn{5}{|c|}{$\begin{array}{c}\text { Mutations in the gyrA gene } \\
\text { at codon positions }\end{array}$} & \multirow{2}{*}{$\begin{array}{l}\text { No mutation } \\
\quad(\mathrm{n}=21)\end{array}$} \\
\hline & & 4 & 8 & 16 & $>16$ & 88 & 90 & 91 & 94 & Double substitutions & \\
\hline OFX monoresistant & 19 & 2 & 5 & 7 & 5 & & 3 & & 13 & 1 & 2 \\
\hline \multicolumn{12}{|l|}{ Polyresistant $(\mathrm{n}=16)$} \\
\hline SM, OFX & 4 & 1 & 2 & 1 & & & 1 & & 2 & & 1 \\
\hline INH, OFX & 6 & 3 & 1 & 1 & 1 & & 1 & & 2 & & 3 \\
\hline RIF, OFX & 2 & 2 & & & & & 2 & & & & \\
\hline INH, EMB, OFX & 1 & & & 1 & & & & & & & 1 \\
\hline SM, INH, OFX & 2 & & 1 & & 1 & & 2 & & & & \\
\hline SM, INH, EMB, OFX & 1 & & & 1 & & & & & 1 & & \\
\hline \multicolumn{12}{|l|}{$M D R(\mathrm{n}=65)$} \\
\hline INH, RIF, OFX & 14 & 1 & 7 & 5 & 1 & & 6 & 1 & 3 & 1 & 3 \\
\hline SM, INH, RIF, OFX & 23 & 1 & 1 & 17 & 4 & 1 & 3 & 1 & 10 & 4 & 4 \\
\hline INH, RIF, EMB, OFX & 6 & 1 & 2 & 3 & & & 1 & & 2 & 1 & 2 \\
\hline Panresistant (SM, INH, RIF, EMB, OFX) & 22 & 6 & 4 & 10 & 2 & & 5 & & 8 & 4 & 5 \\
\hline Total MDR & 65 & 9 & 14 & 35 & 7 & 1 & 15 & 2 & 23 & 10 & 14 \\
\hline Total & 100 & 17 & 23 & 46 & 14 & 1 & 24 & 2 & 41 & 11 & 21 \\
\hline
\end{tabular}

Abbreviations: EMB, ethambutol; INH, isoniazid; LJ, lowenstein jensen; MDR, multidrug resistant; OFX, ofloxacin; RIF, rifampicin; SM, streptomycin. 
Table 2 Mutations in the QRDR of the gyrA and gyrB genes and OFX MIC profile of $M$. tuberculosis

\begin{tabular}{|c|c|c|c|c|c|c|c|c|}
\hline \multirow[b]{2}{*}{ Codon } & \multirow{2}{*}{$\begin{array}{l}\text { No. of OFX-resistant } \\
\text { isolates }(\mathrm{n}=79)\end{array}$} & \multirow[b]{2}{*}{ Nucleotide change } & \multirow[b]{2}{*}{ Amino acid change } & \multicolumn{4}{|c|}{ OFX MIC $\left(\mu g m l^{-1}\right)$} & \multirow[b]{2}{*}{ Frequency $(\%$} \\
\hline & & & & 4 & 8 & 16 & $>16$ & \\
\hline 90 gyrA & 24 & GCG-GTG & Ala90Val & 4 & 8 & 10 & 2 & 24/79 (30.4) \\
\hline 91 gyrA & 2 & TCG-CCG & Ser91Pro & 1 & & 1 & & 2/79 (2.5) \\
\hline \multirow[t]{3}{*}{94 gyrA } & 3 & GAC-CAC & Asp94His & & 1 & 1 & 1 & $3 / 79(3.9)$ \\
\hline & 7 & GAC-GCC & Asp94Ala & & 3 & 4 & & $7 / 79(8.9)$ \\
\hline & 5 & GAC-TAC & Asp94Tyr & 1 & 1 & 3 & & $5 / 79(6.3)$ \\
\hline 90 and 91 gyrA & 4 & $\begin{array}{l}\text { GCG-GTG, } \\
\text { TCG-CCG }\end{array}$ & Ala90Val, Ser91Pro & & 1 & 2 & 1 & $4 / 79(5.1)$ \\
\hline 90 and 94 gyrA & 3 & $\begin{array}{l}\text { GCG-GTG, } \\
\text { GAC-AAC }\end{array}$ & Ala90Val, Asp94Asn & & & 1 & 2 & 3/79 (3.8) \\
\hline 90 gyrA with 539 gyrB & 2 & $\begin{array}{l}\text { GCG-GTG, } \\
\text { ACC-AAC }\end{array}$ & Ala90Val, Thr539ASn & & 2 & & & $2 / 79(2.5)$ \\
\hline 90 gyrA with 500 gyrB & 1 & $\begin{array}{l}\text { GCG-GTG, } \\
\text { GAC-GCC }\end{array}$ & Ala90Val, Asp500Ala & & & 1 & & $1 / 79(1.3)$ \\
\hline 94 gyrA with 592 gyrB & 1 & $\begin{array}{l}\text { GAC-GCC, } \\
\text { CCG-TCG }\end{array}$ & Asp94Ala, Pro592Ser & & 1 & & & $1 / 79(1.3)$ \\
\hline 94 gyrA with 538 gyrB & 1 & $\begin{array}{l}\text { GAC-GGC, } \\
\text { AAC-ATC }\end{array}$ & Asp94Gly, Asn538lle & & 1 & & & $1 / 79(1.3)$ \\
\hline
\end{tabular}

Abbreviations: OFX, ofloxacin; QRDR, quinolone resistance determining region.

the gyrA gene with FQ resistance in M. tuberculosis. ${ }^{2,13}$ The relationship between double mutations in the gyrA gene and the MICs of OFX-resistant M. tuberculosis isolates are not well studied. In our study, the relationship between the OFX MICs and double mutations in the gyrA gene showed no significant association.

Studies have reported a mutation at codon 95 (Ser95Thr) as a natural polymorphism that is not related to FQ resistance. ${ }^{14}$ The frequency of the gyrA gene mutations in this study was found at a lower rate than reported from Russia $(83 \%),{ }^{15}$ but higher than reported from the Guangdong province $(73.3 \%){ }^{3}$ In earlier studies, mutations at codons 90, 91 and 94 were reported $^{2,14,16}$ in FQ-resistant isolates. Matrat et al. ${ }^{17}$ reported less common involvement at codon 88. Studies from Germany, Taiwan, Russia and Kuwait have reported that Asp94Gly is the most common mutation observed in FQresistant M. tuberculosis isolates. ${ }^{15,16}$ Various studies reported double mutations at codons Ala90Val with Asp94Gly, Ala90Val with Ser91Pro and Ala90Val with Asp94Asn ${ }^{18}$ as found in the present study with an another combination, Gly88Ala with Asp94Asn. Some of the OFXresistant isolates $(n=21)$ did not show any mutation in the gyrA gene and may be explained by other mechanisms of FQ drug resistance, such as mutations outside the QRDR, decreased cell permeability of the drug or other mechanisms of resistance, for example, efflux pump. ${ }^{19}$

Studies have reported a small number of FQ-resistant isolates containing mutations in the $\operatorname{gyrB}$ gene. ${ }^{12,15,18}$ Some reported mutations such as Asp500Ala, ${ }^{5}$ Asn538Ile ${ }^{20}$ and Thr539Asn ${ }^{12}$ were identified in OFX-resistant isolates in this study. The Thr539Asn mutation was identified in an OFX-resistant strain from East China, in combination with an Ala90Val gyrA gene mutation, ${ }^{12}$ which is similar to our findings. Moreover, in this study, all of the isolates showing gyrB gene mutations also carried mutations in the gyrA gene. In addition, the gyrB gene mutations were found in a smaller number of cases, and were not frequent enough to evaluate their significance and association with FQ susceptibility.

In conclusion, our findings support previous studies that the OFX resistance to $M$. tuberculosis is associated with mutations in the QRDR of the gyrA gene. However, the level of resistance for OFX-resistant isolates could not be predicted based on the mutation patterns in the gyrA gene.

\section{ACKNOWLEDGEMENTS}

This research work was supported by the Government of India, Ministry of Science and Technology, Department of Science and Technology, New Delhi110016.

1 Cole, S. T. et al. Deciphering the biology of Mycobacterium tuberculosis from the complete genome sequence. Nature 393, 537-544 (1998).

2 Takiff, H. E. et al. Cloning and nucleotide sequence of Mycobacterium tuberculosis gyrA and gyrB genes and detection of quinolone resistance mutations. Antimicrob. Agents Chemother. 38, 773-780 (1994).

3 Yin, X. \& Yu, Z. Mutation characterization of gyrA and gyrB genes in levofloxacinresistant Mycobacterium tuberculosis clinical isolates from Guangdong Province in China. J. Infect. 61, 150-154 (2010).

4 Brossier, F., Veziris, N., Aubry, A., Jarlier, V. \& Sougakoff, W. Detection by GenoType MTBDRsl test of complex mechanisms of resistance to second line drugs and ethambutol in multidrug resistant Mycobacterium tuberculosis complex isolates. J. Clin. Microbiol. 48, 1683-1689 (2010).

5 Duong, D. A. et al. Beijing genotype of Mycobacterium tuberculosis is significantly associated with high-level fluoroquinolone resistance in Vietnam. Antimicrob. Agents Chemother. 53, 4835-4839 (2009). 
6 Canetti, G. et al. Advances in techniques of testing mycobacterial drug sensitivity and the use of sensitivity tests in tuberculosis control programmes. Bull. WHO. 41, 21-43 (1969).

7 Aslan, G., Tezcan, S., Serin, M. S. \& Emekdas, G. Genotypic analysis of isoniazid and rifampin resistance in drug-resistant clinical Mycobacterium tuberculosis Complex isolates in Southern Turkey. Jpn J. Infect. Dis. 61, 255-260 (2008).

8 Singh, M. et al. In vitro effect of fluoroquinolones against Mycobacterium tuberculosis isolates from Agra \& Kanpur region of north India. Indian J. Med. Res. 542-547 (2009).

9 Verma, J. S., Nair, D., Rawat, D. \& Manzoor, N. Assessment of trends of ofloxacin resistance in Mycobacterium tuberculosis. Indian J. Med. Res. 29, 280-282 (2011).

10 Daniel, O. et al. Ofloxacin resistance among Mycobacterium tuberculosis isolates in two states of south-west Nigeria. Afr. J. Resp. Med. 6, 18-20 (2011).

11 Umubyeyi, A. N. et al. Limited fluoroquinolone resistance among Mycobacterium tuberculosis isolates from Rwanda: results of a national survey. J. Antimicrob. Chemother. 59, 1031-1033 (2007).

12 Cui, Z., Wang, J., Lu, J., Huang, W. \& Hu, Z. Association of mutation patterns in gyrA/B genes and ofloxacin resistance levels in Mycobacterium tuberculosis isolates from East China in 2009. BMC Infect. Dis. 11, 78 (2011).

13 Chen, J. et al. Characterization of gyr $A$ and gyrB mutations and fluoroquinolone resistance in Mycobacterium tuberculosis clinical isolates from Hubei Province, China. Braz. J. Infect. Dis. 16, 136-141 (2012).
14 Siddiqi, N. et al. Molecular characterization of multidrug-resistant isolates of Mycobacterium tuberculosis from patients in North India. Antimicrob. Agents Che mother. 46, 443-450 (2002).

15 Mokrousov, I. et al. Molecular characterization of ofloxacin-resistant Mycobacterium tuberculosis strains from Russia. Antimicrob. Agents Chemother. 52, 2937-2939 (2008).

16 Al-Mutairi, N., Ahmad, S. \& Mokaddas, E. Performance comparison of four methods for rapid detection of multidrug-resistant Mycobacterium tuberculosis strains. Int. J. Tuberc. Lung Dis. 15, 110-115 (2011).

17 Matrat, S. et al. Functional analysis of DNA gyrase mutant enzymes carrying mutations at position 88 in the A subunit found in clinical strains of Mycobacterium tuberculosis resistant to fluoroquinolones. Antimicrob. Agents Chemother. 50, 4170-4173 (2006)

18 Von Groll, A. et al. Fluoroquinolone resistance in Mycobacterium tuberculosis and mutations in gyrA and gyrB. Antimicrob. Agents Chemother. 53, 4498-4500 (2009).

19 Escribano, I. et al. Importance of the efflux pump systems in the resistance of Mycobacterium tuberculosis to fluoroquinolones and linezolid. Chemotherapy 53, 397-401 (2007).

20 Devasia, R. et al. High proportion of fluoroquinolone resistant Mycobacterium tuberculosis isolates with novel gyrase polymorphisms and a gyrA region associated with fluoroquinolone susceptibility. J. Clin. Microbiol. 50, 1390 (2012). 